\title{
Mixed tuberculosis infections in Northwest of Iran
}

\author{
Mohammad Asgharzadeh'1, Zahra Taghinejad², Behroz Mahdavipoor ${ }^{3}$, \\ Vahid Asgharzadeh ${ }^{4}$, Hossein Samadi Kafil ${ }^{5}$, Jalil Rashedi ${ }^{6}$ \\ ${ }^{1}$ Biotechnology Research Center and Faculty of Paramedicine, Tabriz University of Medical Sciences, Tabriz, Iran; \\ ${ }^{2}$ Hematology and Oncology Research Center, Tabriz University of Medical Sciences, Tabriz, Iran; \\ ${ }^{3}$ Department of Laboratory Sciences, Faculty of Paramedicine, Tabriz University of Medical Sciences, Tabriz, Iran; \\ ${ }^{4}$ Student Research Committee, Tabriz University of Medical Sciences, Tabriz, Iran; \\ ${ }^{5}$ Drug Applied Research Center, Tabriz University of Medical Sciences, Tabriz, Iran; \\ ${ }^{6}$ Tuberculosis and Lung Disease Research Center, Tabriz University of Medical Sciences, Tabriz, Iran
}

Article received 23 August, 2021; accepted 17 October, 2021

\section{SUMMARY}

Primary Mycobacterium tuberculosis (MTB) infection doesn't provide protection against secondary infection and patients can suffer from multiple strains of $M$. tuberculosis simultaneously. The aim of this study was to use molecular genotyping to identify cases of mixed infection in Northwest of Iran.

One hundred and twenty-one positive culture isolates of $M$. tuberculosis were prepared from patients consecutively in Northwest of Iran from March 2017 to March 2018 and then microevolution and mix infection were assessed using the mycobacterial interspersed repetitive unit-variable number tandem repeat (MIRU-VNTR) method.
MIRU-VNTR analysis revealed that nine samples $(7.3 \%)$ had double alleles in at least one locus, as observed by five cases of microevolution, and four cases $(3.3 \%)$ of mixed infection.

According to this study, mixed infection in Northwest of Iran has significantly decreased compared to 13 years ago $(7.1 \%$ decreased to $3.3 \%)$, and in order to eradicate tuberculosis it is necessary to identify all cases of mixed infection, at least in recurrent cases, in the future.

Keywords: Mycobacterium tuberculosis, mixed infection, microevolution, MIRU-VNTR, Iran.

\section{INTRODUCTION}

$\mathrm{T}$ uberculosis (TB) is an ancient disease, as the effects of TB tissue damage can be observed in the bones of Egyptian mummified corpses. It is claimed that it has caused more deaths than any other microbial pathogen [1]. Today in spite of years of anti-TB drugs' discovery, widespread usage of the Bacillus Calmette-Guérin (BCG) vaccine and different diagnostic methods, as well as the adoption of Directly Observed Therapy Shortcourse (DOTs) for disease control, TB remains a major health problem worldwide [2]. The num-

Corresponding author

Jalil Rashedi

E-mail: Rashedijalil@gmail.com ber of individuals infected with TB is so high that over 10 million new cases occurred worldwide in 2019, and about 1,408,000 people died of TB, of whom 208,000 were HIV-positive [3]. It has been demonstrated that primary infection cannot provide protection against secondary infection and TB-infected patients can be infected with more than one strain of MTB $[4,5]$. MTB infection can even occur in previously TB-infected patients, during or after successful treatment [6].

For more than two decades, molecular techniques including IS6110-RFLP[7], spoligotyping, and MIRU-VNTR have been used to study molecular diversity, to determine recent transmission, to identify TB recurrence due to reinfection from relapse and cross-contamination [8-10]. Additionally, the methods can also identify co-infection with multiple different strains of MTB [4, 11, 12]. MIRU- 
VNTR is one of the most effective techniques for identifying mixed infections [13]. MIRU-VNTR identifies sequences that repeat with various copies in different MTB. It differentiates mixed infection from microevolution. In mixed infection there is more than one allele in at least two MIRU Loci, while in microevolution two alleles are detected in only one locus [11, 14]. MIRU-VNTR has $2,4,10,16,20,23,24,26,27,31,39,40, \mathrm{~A}, \mathrm{~B}, \mathrm{C}$ loci with good diversity that are suitable for examining mixed infection [9]. Mixed infection is one of the obstacles to comprehensive TB control and eradication because it is not easy to diagnose drug resistance when a patient is infected with both sensitive and resistant strains [15]. Understanding the relative percentage of mixed MTB infections is critical to facilitate the prevention of the spread of drug-resistant strains, evaluation of new drugs and interpretation of molecular epidemiological data $[12,13,16]$. So as a part of taking more accurate preventive decisions to reduce TB in East Azerbaijan province, Iran, it was decided to use MIRU-VNTR to determine the prevalence of mixed MTB infections in this region.

\section{MATERIALS AND METHODS}

\section{Mycobacterial isolates}

Mycobacterium tuberculosis isolates were obtained from all patients referred to the Central Tuberculosis Laboratory in northwestern Iran from March 2017 to March 2018. The study included patients with at least one positive sample for MTB. Totally, 121 isolates of MTB were collected and identification of mycobacterium isolates was performed by using Ziehl-Neelsen staining and standard microbiological tests like niacin production capability, catalase activity, nitrate reconstruction, pigment production and growth rate in the Lowenstein Jensen Medium [17].

\section{MIRU-VNTR}

DNA from mycobacterial isolates was extracted by using lysozyme, SDS (sodium dodecyl sulfate), proteinase $\mathrm{K}$ and $\mathrm{CTAB}$ (cetyl trimethyl ammonium bromide) and then dissolved in distilled water following precipitation with isopropanol and washing with $70 \%$ ethanol [18]. PCR was performed in the volume of $20 \mu$ l containing 10-100 ng of DNA, $0.5 \mu \mathrm{M}$ of specific primers, $1.5 \mathrm{mM}$ of $\mathrm{MgCl} 2,100 \mu \mathrm{M}$ of dATP, dTTP, dCTP, dGTP, and
$50 \mathrm{mM}$ of $\mathrm{KCl}, 20 \mathrm{mM}$ of Tris- $\mathrm{Cl}(\mathrm{pH}=8), 1.25 \mathrm{U}$ recombinant Taq DNA polymerase (Sinaclon Co, Iran) and then $40 \mu \mathrm{l}$ sterile paraffin was poured [9]. PCR was done with initial $7 \mathrm{~min}$ denaturation step at $94^{\circ} \mathrm{C}$ and a final 7 min extension step at $72^{\circ} \mathrm{C}$. The temperature cycles for different types of PCRs were as follow, 35 cycles of $1 \mathrm{~min}$ at $95^{\circ} \mathrm{C}$, annealing temperature for $1 \mathrm{~min}$ and $72^{\circ} \mathrm{C}$ for 1 min. Annealing temperatures were used as follows: $65,63,68,65,59,65,59,64,64,63,68,65,66$, 66, and 66 for MIRU-VNTR loci 2, 4, 10, 16, 20, 23, $24,26,27,31,39,40$, A, B, and C, respectively. Cycling was carried out in Cyclogene thermal cycler of Techne Company.

In all the PCRs, the negative controls consisted of the PCR components in reaction mixtures lacking bacterial DNA. PCR products were electrophoresed in $1.5 \%$ agarose gel and after staining with DNA safe stain visualized under UV light. The size of fragments was determined in comparing with 100bp DNA ladder plus size marker (Fermentas, Lithuania).

\section{RESULTS}

The mixed infection was seen in 4 of 121 isolated samples from TB patients. These 4 samples (3.3\%) had 2 to 3 alleles in two or more loci. MIRU-VNTR analysis also indicated that at least 9 of the 121 samples $(7.3 \%)$ contained double alleles in at least one locus and thatmicroevolution was present in 5 loci, implying that only two alleles were found in one locus (Table 1). In this study, out of 15 loci, there were seven loci with multiple alleles including, loci 40 (four cases), 26 (three cases), 10 (two cases), 16 (two cases), B (two cases), and 31, and A locus each having one multiple allele. Meanwhile in the previous study, 12 multiple alleles were observed in 15 loci of 154 samples.

\section{DISCUSSION}

The goal of TB control programs is to eradicate the disease by breaking the transmission chain, which is effectively possible with rapid diagnosis and definitive treatment of active TB cases [19]. Primary infection, reactivation of primary infection, exogenous re-infection with a new strain of MTB, and mixed infection are all causes of active TB. Detection of microevolution and also mixed infection is possible through fingerprinting, especially 
Table 1 - Isolates showing multiple allele for one or more VNTR Ioci.

\begin{tabular}{|c|c|c|c|c|c|c|c|c|c|c|c|c|c|c|c|}
\hline \multirow{2}{*}{ Strain ID } & \multicolumn{15}{|c|}{ allele(s) at MIRU-VNTR locus } \\
\hline & 2 & 4 & 10 & 16 & 20 & 23 & 24 & 26 & 27 & 31 & 39 & 40 & $A$ & $B$ & C \\
\hline \multicolumn{16}{|l|}{ Mixed } \\
\hline 36 & 2 & 3 & 5 & 3,4 & 2 & 5 & 2 & 2,7 & 3 & 3 & 2 & 3 & 4 & 2 & 2 \\
\hline 60 & 2 & 3 & 3,5 & 1 & 2 & 5 & 2 & 5 & 3 & 3 & 2 & 2,3 & 2 & 4 & 4 \\
\hline 82 & 2 & 3 & 2,3 & 3 & 2 & 5 & 2 & 5 & 3 & $0,3,4$ & 2 & $2,3,4$ & 3 & 2,4 & 4 \\
\hline 93 & 2 & 3 & 4 & 3 & 2 & 5 & 1 & 3,5 & 3 & 3 & 2 & 2 & 3 & 1,2 & 3 \\
\hline \multicolumn{16}{|c|}{ Microevolution } \\
\hline 24 & 2 & 2 & 3 & 3 & 1 & 5 & 2 & 4 & 3 & 3 & 2 & 4 & 3,4 & 2 & 4 \\
\hline 31 & 2 & 3 & 3 & 3 & 2 & 6 & 2 & 3,5 & 3 & 3 & 2 & 2 & 2 & 2 & 5 \\
\hline 73 & 2 & 3 & 3 & 3 & 2 & 5 & 2 & 7 & 3 & 4 & 2 & 3,4 & 4 & 2 & 4 \\
\hline 77 & 2 & 3 & 3 & 1 & 2 & 5 & 2 & 5 & 2 & 3 & 2 & 2,4 & 3 & 1 & 4 \\
\hline 112 & 2 & 3 & 3 & 2,3 & 2 & 5 & 1 & 5 & 3 & 3 & 2 & 2 & 3 & 2 & 4 \\
\hline
\end{tabular}

MIRU-VNTR methods [11-13]. In this study, microevolution was detected in $4.1 \%$ of the samples, which shows a little reduction compared with the prior study performed in Northwest of Iran in which the microevolution was $6.5 \%$ (unpublished data). Observed microevolution in current study is also lower than microevolution in Eastern Europe $(7.3 \%)$ and Africa (7\%) [11, 15]. Microevolution can occur during the latency phase, active phase of the disease, and logarithmic growth phase during culture and it can impact any genetic marker that affects the expression of neighboring genes [20]. Moreover, microevolution can impact TB transmission chain through increasing MTB proliferation within macrophages [21]. In this study, 3.3\% of cases (4 samples) had 2 or more than 2 alleles in two or more loci that in one case two or more alleles were observed in 4 loci which means that the patient was infected with 3 distinct MTB strains (Table 1). Similar to our study mixed infection was $3.1 \%$ in Inner Mongolia, China, and Vietnam [22, 23]. However, mixed infection was lower than our study in Guyana and Suriname $(0.6 \%)$, Botswana $(1.7 \%)$, Sichuan province, China (2.4\%), and Florida, USA (2.6\%) [12, 14, 24, 25]. Mixed infection in Kampala, Uganda (7.1\%), South Africa (9\%), Georgia (13.1\%), the capital of Iran $(26.6 \%)$, and Tehran province, Iran (53\%) was higher than in our study [5, 11, 26-28]. In our opinion, the high frequency of mixed infections in these studies is related to the high prevalence of HIV positive individuals, including the samples from patients died in the area with a high prevalence of HIV, hospitalized prisoners, and patients with a history of TB with various antibiotic resistance patterns, as well as having been carried on in a congested city with a large number of Afghan immigrants likewise immigrants play a critical role in TB transmission and increase the disease's cases [5, 11, 26-30].

Allele frequency was four in locus 26, a figure higher than the other loci. Loci 40, 16, B, 10, 31, $\mathrm{A}, 23,27, \mathrm{C}, 39$, and 4 were in the next ranks, respectively (unpublished data), so loci $40,26,10$, 16, B, 31 and A MIRU-VNTR typing can be utilized in Iran, especially in Northwest, to analyze mixed infections quickly. In the current study mixed infections were $3.3 \%$, which shows a significant reduction compared with the prior study with $7.1 \%$ mixed infection frequency. This significant reduction is due to consideration of directly observed therapy, free treatment of TB patients at $\mathrm{TB}$ diagnosis and treatment centers, performing molecular epidemiologic studies in Northwest region of Iran and isolating hospitalization place of multi drug resistance TB (MDR-TB) patients from non-MDR-TB in the city of Tabriz, the capital of the province. To control the tuberculosis, it is necessary to pay special attention to the MDR-TB, especially in patients with mixed infections [31]. Treatment failure in TB patients is caused by a variety of factors, including incomplete drug administration, irregular drug use, premature drug discontinuation, drug resistance, and mixed in- 
fection, particularly when the patient is infected with a mix of resistant and sensitive microorganisms and drug resistance is not correctly diagnosed. Antibiotic treatment pressure can alter the dominant strains in sputum culture, resulting in treatment failure and increased mortality in patients with mixed infections, thus patients should be given extra care $[6,25,32]$. Furthermore, as mixed infections accelerate the propagation of drug-resistant strains, drug susceptibility testing on various colonies is essential in mixed infections [6, 27]. In detecting mixed infections, the quality of sputum, testing on sputum samples, culture medium, minimum number of cultures, molecular typing method and infection status can be effective [33]. Acquisition of mixed infections depends on the incidence of infection in the community and the diversity of its strains found in the environment and population, but it can occur in places where the prevalence of tuberculosis is low [23, 25]. In jails and regions where HIV is more prevalent, mixed infection is widespread $[11,26]$. Local TB frequency, past tuberculosis treatment history, long-term hospitalization, aging, congenital immunodeficiency, and immunosuppressive conditions including alcoholism, malnutrition, renal failure, cancer, children suffering from rheumatoid arthritis, leukemia and diabetes can all raise the likelihood of mixed infection [25, 28, 34-36]. To lessen the amount of instances of mixed infection, it is important to treat latent TB infections and halt transmission, which may be accomplished by speeding up access to high-risk individuals such as HIV-positive people and identifying suspects [24].

There is a risk of false positives in mixed infections due to cross-contamination. So, in the mycobacteriology laboratory attention should be paid to good laboratory practice, such as preparing appropriate questionnaire for each sample submitted to the laboratory, processing at least one negative control sample per working day, preventing aerosols creation and contamination in performing MIRU-VNTR to minimize the risk of cross contamination [37]. As spoligotyping and IS6110-RFLP are unable to detect mixed strains of a lineage, the MIRU-VNTR method outperforms them in detecting mixed infection. Although spoligotyping is less sensitive and certain strains lack the IS6110 gene, the MIRU-VNTR may distinguish several strains of the same lineage [38,
39]. However, as MIRU-VNTR only detects differences in a few loci, employment of the wholegenome-sequencing (WGS) would be excellent for identifying strains and instances of mixed infection. Of course, this approach also has limitations of reading duplicate regions, requiring complex data analysis, and high cost [20]. It is critical to detect mixed infection since ignoring it might increase TB mortality and postpone eradication. In this study, there were the following limitations: 1) this study included only a limited number of patients whose samples were sent to a reference laboratory.

2) MIRU-VNTR was carried out with 15 loci.

3) Each person had only one culture sample analyzed.

4) The impact of risk factors has not been studied due to the limited number of mixed infections. It can be concluded that $3.3 \%$ of TB patients had mixed infection, which has been significantly decreased compared to the previous study $(7.1 \%)$. This reduction would be owing to improved diagnostic methods and directly observed therapy. Eventually for eradicating TB, all cases of mixed infection, or at least samples of recurrent patients, are recommended to be identified through molecular typing.

\section{Ethical considerations}

Ethical issues (plagiarism, informed consent, misconduct, data fabrication and/or falsification, double publication and/or submission, redundancy, etc.) have been completely observed by the authors.

\section{Acknowledgements}

We thank of Tabriz TB center for their generous cooperation.

\section{Conflict of interest}

All of the authors declare that they have no conflict of interest.

\section{Funding declaration}

This study was supported by Tabriz University of Medical Sciences (grant number: 65019).

\section{REFERENCES}

[1] Daniel TM. The history of tuberculosis. Respir Med. 2006; 100 (11), 1862-70. 
[2] Colomba C, Rubino R, Mantia G, et al. Clinical use of BCG and its complications: a case series. Infez Med. 2021; 29 (1), 123-9.

[3] WHO G. Global tuberculosis report 2020. Geneva: World Health Organization; 2020. Licence: CC BY-NCSA 3.0 IGO.: https:/ /WWW.Who.int/publications/lilitem/9789240013131.; 2020.

[4] Warren RM, Victor TC, Streicher EM, et al. Patients with active tuberculosis often have different strains in the same sputum specimen. Am J Respir Crit Care Med. 2004; 169 (5), 610-4.

[5] Dickman KR, Nabyonga L, Kateete DP, et al. Detection of multiple strains of Mycobacterium tuberculosis using MIRU-VNTR in patients with pulmonary tuberculosis in Kampala, Uganda. BMC Infect Dis. 2010; 10 (1), 349.

[6] Van Rie A, Victor TC, Richardson M, et al. Reinfection and mixed infection cause changing Mycobacterium tuberculosis drug-resistance patterns. Am J Respir Crit Care Med. 2005; 172 (5), 636-42.

[7] Asgharzadeh M, Kafil HS, Roudsary AA, et al. Tuberculosis transmission in Northwest of Iran: Using MIRU-VNTR, ETR-VNTR and IS6110-RFLP methods. Infect Genet Evol. 2011; 11 (1), 124-31.

[8] Asgharzadeh M, Rashedi J, Poor BM, et al. How molecular epidemiology can affect tuberculosis control in the Middle East Countries: a systematic review and meta-analysis. Infect Disord Drug Targets. 2021; 21 (1), 28-37. [9] Supply P, Allix C, Lesjean S, et al. Proposal for standardization of optimized mycobacterial interspersed repetitive unit-variable-number tandem repeat typing of Mycobacterium tuberculosis. J Clin Microbiol. 2006; 44 (12), 4498-510.

[10] Barac A, Karimzadeh-Esfahani H, Pourostadi M, et al. Laboratory cross-contamination of Mycobacterium tuberculosis: a systematic review and meta-analysis. Lung. 2019; 197 (5), 651-61.

[11] Cohen T, Wilson D, Wallengren K, et al. Mixedstrain Mycobacterium tuberculosis infections among patients dying in a hospital in KwaZulu-Natal, South Africa. J Clin Microbiol. 2011; 49 (1), 385-8.

[12] Zheng C, Li S, Luo Z, et al. Mixed infections and rifampin heteroresistance among Mycobacterium tuberculosis clinical isolates. J Clin Microbiol. 2015; 53 (7), 2138-47.

[13] Tarashi S, Fateh A, Mirsaeidi M, et al. Mixed infections in tuberculosis: The missing part in a puzzle. Tuberculosis (Edinb). 2017; 107, 168-74.

[14] Streit E, Millet J, Rastogi N. Mycobacterium tuberculosis polyclonal infections and microevolution identified by MIRU-VNTRs in an epidemiological study. Int $J$ Mycobacteriol. 2015; 4 (3), 222-7.

[15] Kontsevaya I, Nikolayevskyy V, Kovalyov A, et al. Tuberculosis cases caused by heterogeneous infection in Eastern Europe and their influence on outcomes. Infect Genet Evol. 2017; 48, 76-82.
[16] Cohen T, van Helden PD, Wilson D, et al. Mixedstrain Mycobacterium tuberculosis infections and the implications for tuberculosis treatment and control. Clin Microbiol Rev. 2012; 25 (4), 708-19.

[17] Rieder HL, Chonde TM, Myking H, et al. The public health service national tuberculosis reference laboratory and the national laboratory network; minimum requirements, role and operation in a low-income country: International Union Against Tuberculosis And Lung Disease (IUATLD). 1998.

[18] Asgharzadeh M, Kafil HS, Khakpour M. Comparison of mycobacterial interspersed repetitive unit-variable number tandem repeat and IS6110-RFLP methods in identifying epidemiological links in patients with tuberculosis in Northwest of Iran. Ann Microbiol. 2008; 58 (2), 333-9.

[19] Pasticci MB, Papalini C, Murgia N, et al. QuantiFERON-TB and tuberculin skin test in patients with active tuberculosis: the experience of a single medium-sized Italian University Hospital. Interferon. 2021; 1, 11-13.

[20] Ford CB, Lin PL, Chase MR, et al. Use of whole genome sequencing to estimate the mutation rate of Mycobacterium tuberculosis during latent infection. Nat Genet. 2011; 43 (5), 482-6.

[21] Pérez-Lago L, Herranz M, Martinez Lirola M, et al. Characterization of microevolution events in Mycobacterium tuberculosis strains involved in recent transmission clusters. J Clin Microbiol. 2011; 49 (11), 3771-6.

[22] Wang X, Liu H, Wei J, et al. An investigation on the population structure of mixed infections of Mycobacterium tuberculosis in Inner Mongolia, China. Tuberculosis. 2015; 95 (6), 695-700.

[23] Huyen MN, Kremer K, Lan NT, et al. Mixed tuberculosis infections in rural South Vietnam. J Clin Microbiol. 2012; 50 (5), 1586-92.

[24] Baik Y, Modongo C, Moonan PK, et al. Possible transmission mechanisms of mixed Mycobacterium tuberculosis infection in high HIV prevalence country, Botswana. Emerg Infect Dis. 2020; 26 (5), 953.

[25] Asare-Baah M, Séraphin MN, Salmon LA, et al. Effect of mixed strain infections on clinical and epidemiological features of tuberculosis in Florida. Infect Genet Evol. 2021; 87, 104659.

[26] Shamputa IC, Jugheli L, Sadradze N, et al. Mixed infection and clonal representativeness of a single sputum sample in tuberculosis patients from a penitentiary hospital in Georgia. Respir Res. 2006; 7 (1), 1-10.

[27] Hajimiri ES, Masoomi M, Ebrahimzadeh N, et al. High prevalence of Mycobacterium tuberculosis mixed infection in the capital of moderate tuberculosis incidence country. Microb Pathog. 2016; 93, 213-8.

[28] Kamakoli MK, Sadegh HR, Farmanfarmaei G, et al. Evaluation of the impact of polyclonal infection and heteroresistance on treatment of tuberculosis patients. Scientific Reports. 2017; 7 (1), 1-4.

[29] Mohammadzadeh A, Rashedi J, Poor BM, et al. 
Role of immigration in tuberculosis transmission to Iran: A systematic review. Int J Prev Med. 2020; 11, 200. doi: 10.4103/ijpvm.IJPVM_463_18.

[30] Scotto G, Fazio V, Lo Muzio L. Tuberculosis in the immigrant population in Italy: state-of-the-art review. Infez Med. 2017; 25 (3), 199-209.

[31] Kazemian H, Kardan-Yamchi J, Mosavari N, et al. Molecular characterization of multidrug and extensive drug-resistant Mycobacterium tuberculosis isolates from Iran. Infez Med. 2019; 27 (1), 26-31.

[32] Zetola NM, Modongo C, Moonan PK, et al. Clinical outcomes among persons with pulmonary tuberculosis caused by Mycobacterium tuberculosis isolates with phenotypic heterogeneity in results of drug-susceptibility tests. J Infect Dis. 2014; 209 (11), 1754-63.

[33] McIvor A, Koornhof H, Kana BD. Relapse, re-infection and mixed infections in tuberculosis disease. Pathog Dis. 2017; 75 (3).

[34] Shin SS, Modongo C, Ncube R, et al. Advanced immune suppression is associated with increased prevalence of mixed-strain Mycobacterium tuberculosis infections among persons at high risk for drug-resistant tu- berculosis in Botswana. I Infect Dis. 2015; 211 (3), 347-51. [35] Ozma MA, Rashedi J, Poor BM, et al. Tuberculosis and diabetes mellitus in Northwest of Iran. Infect Disord Drug Targets. 2020; 20 (5), 667-71.

[36] Bruzzese E, Bocchino M , Assante LR, et al. Gamma interferon release assays for diagnosis of tuberculosis infection in immune-compromised children in a country in which the prevalence of tuberculosis is low. J Clin Microbiol. 2009; 47 (7), 2355-7.

[37] Asgharzadeh M, Ozma MA, Rashedi J, et al. Falsepositive Mycobacterium tuberculosis detection: Ways to prevent cross-contamination. Tuberc Respir Dis. 2020; 83 (3), 211-17.

[38] Joseph BV, Soman S, Radhakrishnan I, et al. Molecular epidemiology of Mycobacterium tuberculosis clinical isolates from Kerala, India using IS6110-RFLP, spoligotyping and MIRU-VNTR typing on stored isolates. Infect Genet Evol. 2013; 16, 157-64.

[39] Martín A, Herránz M, Serrano MJR, et al. Rapid clonal analysis of recurrent tuberculosis by direct MIRU-VNTR typing on stored isolates. BMC Microbiol. 2007; 7 (1), 1-7. 